\title{
Kalıtsal Faktör VIII Eksikliği Hastalarında Rekombinan FVIII'in Farmakokinetik Verileri
}

\section{Pharmacokinetic Studies of Recombinant FVIII in Hereditary Factor VIII Deficiency Patients}

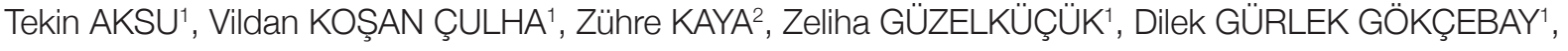
H.Neşe YARALI', Namık Yaşar ÖZBEK'1

'Sağlık Bilimleri Üniversitesi, Ankara Çocuk Sağlığı ve Hastalıkları Hematoloji Onkoloji Sağlık Uygulama Araştırma Merkezi, Çocuk Hematoloji Onkoloji Kliniği, Ankara, Türkiye

${ }^{2}$ Gazi Üniversitesi Tıp Fakültesi, Çocuk Sağlı̆ıı ve Hastalıkları Anabilim Dalı, Çocuk Hematoloji Bilim Dalı, Ankara, Türkiye

\section{ÖZ}

Amaç: Hemofili A hastalarında kanamaların önlenmesi ve kas-iskelet sistemi fonksiyonlarının korunması amacıyla faktör VIII (FVIII) ile profilaksi önerilmektedir. Farmakokinetik (FK) verilerle düzenlenen profilaksi, kanamalardan korunmada ve tedavi maliyetinin azaltımasında günümüzdeki en başarılı stratejidir.

Gereç ve Yöntemler: Bu çalışmada Bayesian yöntemi kullanan, myPKFiT web erişimli program aracılığı ile FVIII FK verileri hesaplanan 17 ağır hemofili A (ortanca yaş 11.5 yıl, ortanca kullandıkları faktör miktarı $25 \mathrm{U} / \mathrm{kg}$ ) hastası sunuldu.

Bulgular: Çalışmaya dahil edilen hastaların ortalama FVIII yarılanma ömrü 9.1 saat (min-max: 6.8-11.4), ortalama FVIII klerensi 4.7 ml/saat/kg (min-max: 2.6-6.8), ortalama FVIIl'in <\%1'e kadar geçen süre ise 47 saat (min-max: 33-61) olarak bulundu. Hasta yaşlarının artmasıyla FVIII yarılanma ömrünün arttığı görüldü. Hastaların hedef FVIII çukur değeri \%1 üzeri olacak şekilde haftalık doz ve takvim simulasyonu uygulandığında 3 hastanın kullandığı FVıII dozunun yeterli olduğu, 5 hastada FVIII dozunun yüksek olduğu, 9 hastanın ise kullandığı FVIII dozunun yeterli olmadığı görüldü.

Sonuç: Çalışmamızda FVIII FK verileri ile profilaksi doz ve sıklıkları düzenlendi.

Anahtar Sözcükler: Bayesian yöntemi, Farmakokinetik, Hemofili A, Profilaksi

\begin{abstract}
Objective: Factor VIII (FVIII) prophylaxis is now considered a gold standard for the treatment of hemophilia A patients to prevent bleeding and maintain musculoskeletal function. FVIII prophylaxis, tailored with pharmacokinetic data, is the most successful approach in preventing bleeding and reducing the cost of treatment.

Material and Methods: In this study, 17 patients with severe hemophilia A (median age 11.5 years, median factor 25 $\mathrm{U} / \mathrm{kg}$ ) whose FVIII pharmacokinetic data were obtained through the myPKFiT web access program, were presented.

Results: The mean FVIII half-life of the patients was 9.1 hours (min-max: 6.8-11.4); the mean FVIII clearance was 4.7 $\mathrm{ml} /$ hour/kg (min-max: 2.6-6.8); and the mean duration of FVIII levels under 1\% was 47 hours (min-max: 33-61). The
\end{abstract}

AKSU T

KOȘAN ÇULHA V

KAYAZ

GÜZELKÜCÜKK Z

GÜRLEK GÖKÇEBAY D

YARALI HN

ÖZBEK NY
Çıkar Çatışması / Conflict of Interest: Tüm yazarlar adına, sorumlu yazar çıkar çatışması olmadığını belirtir.

0000-0003-4968-109X Etik Kurul Onayı / Ethics Committee Approval: Bu çalıșmada ulusal ve uluslararası etik kurallara uyulmuștur. Çalıșma için Ankara Cocuk Sağlığı ve 0000-0003-0729-1202 Hastalıkları Hematoloji Onkoloji Sağlık Uygulama Araștırma Merkezi Klinik Araștırmalar Etik Kurul'undan onay alındı (Karar no: 2018-160). Kayıt sırasında 0000-0002-3798-7246 veliler tarafından araştırmaya katıım için bilgilendirilmiş bir onay imzalanmıștır.

0000-0003-1462-6867 Yazarların katkısı / Contribution of the Authors: AKSU T: Araștırma ve/veya makalenin hipotezini veya fikrini olușturan, sonuçlara ulașmak için

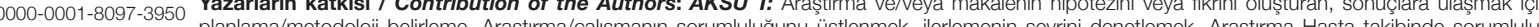
0000-0001-5488-2385 planlama/metodoloji belirleme, Araştırma/çalşmanin sor

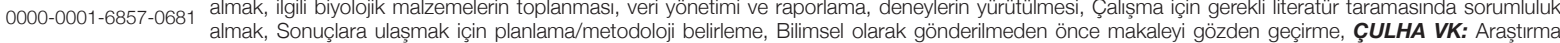
almak, Sonuçlara ulașmak için planlama/metodoloji belirleme, Bilimsel olarak gönderimeden once makaleyi gozden geçirme, ÇULHA VK: Araştırma
Hasta takibinde sorumluluk almak, ilgili biyolojik malzemelerin toplanması, veri yönetimi ve raporlama, deneylerin yürütülmesi, Sonuçlara ulaşmak için planlama/metodoloji belirleme, Bilimsel olarak gönderilmeden önce makaleyi gözden geçirme, KAYA Z: Araştırma Hasta takibinde sorumluluk almak, ilgill biyolojik malzemelerin toplanması, veri yönetimi ve raporlama, deneylerin yürütülmesi, GÜZELKÜÇÜK Z: Araștırma Hasta takibinde sorumluluk almak, ilgili biyolojik malzemelerin toplanması, veri yönetimi ve raporlama, deneylerin yürütülmesi, GÖKÇEBAY DG: Çalıșma için gerekli literatür taramasında sorumluluk almak, YARALI N: Sonuçlara ulașmak için planlama/metodoloji belirleme, Bilimsel olarak gönderilmeden önce makaleyi gözden geçirme, ÖZBEK NY: Araștırma ve/veya makalenin hipotezini veya fikrini olușturan, sonuçlara ulașmak için planlama/metodoloji belirleme, Araștırma/çalıșmanın sorumluluğunu üstlenmek, ilerlemenin seyrini denetlemek, Bilimsel olarak gönderilmeden önce makaleyi gözden geçirme.

Atıf yazım şekli / How to cite : Aksu T, Koșan Çulha V, Kaya Z, Güzelküçük Z, Gürlek Gökçebay D, Yaralı HN ve ark. Kalıtsal Faktör VIII Eksikliğ Hastalarında Rekombinan FVIII'in Farmakokinetik Verileri. Türkiye Çocuk Hast Derg 2020;14:408-412.
Yazıșma Adresi / Correspondence Address:

\section{Tekin AKSU}

Sağlık Bilimleri Üniversitesi, Ankara Çocuk Sağlığı ve Hastalıkları Hematoloji Onkoloji Sağlık Uygulama

Araştırma Merkezi, Çocuk Hematoloji Onkoloji Kliniği, Ankara, Türkiye

E-posta: tekinaksu@gmail.com
Geliş tarihi / Received : 27.06.2020 Kabul tarihi / Accepted : 19.08.2020 Elektronik yayın tarihi $\quad: 16.09 .2020$ Online published

DOI: 10.12956/tchd.758910 
half-life of FVIII was increased with the age of the patient. When a weekly dose schedule simulated with the target FVIII trough level above $1 \%$, it was observed that the dose of FVIII was sufficient in three, it was high in five, and it was insufficient in nine patients.

Conclusion: In our study, prophylaxis doses and frequencies were adjusted according to the FVIII pharmacokinetic data.

Key Words: Hemophilia A, Pharmacokinetic data, Prophylaxis, The Bayesian method

\section{GiRiş}

Hemofili A [kalıtsal Faktör VIII (FVIII) eksikliği], kas ve eklem içi kanamalar, ürogenital, gastrointestinal ve santral sinir sistemi kanamaları ile seyreden nadir bir hastalıktır. Plazmada FVIII düzeyinin \%1'den az olması ağır, \%1-5 arasında orta, \%5 üstü olması hafif hemofili A olarak sınılandırımaktadır (1). Eksik olan FVIII'in kanama durumunda yerine konmasiyla tedavi edilmektedir. Ancak, kanamaların önlenmesi ve kas-iskelet sistemi fonksiyonlarının korunması için FVIII konsantresi ile profilaksi altın standarttır. Üç yaştan önce, ikinci büyük eklem içi kanamadan önce ve klinik veya radyolojik osteokondral eklem hasarı gelișmeden profilaksi bașlanması primer profilaksi kabul edilir (1). İki veya daha fazla büyük eklem kanamasından sonra ve eklem hasarı gelişmeden başlanan sürekli faktör tedavisi ise sekonder profilaksidir (1). Ağır hemofili hastaları için güncel rehberlerde primer profilaksi uygulaması önerilmektedir (1). Profilaksi uygulaması hastanın yaşına, kilosuna, FVIII toparlanma zamanına [TZ (in-vivo recovery)], kanama sıklığına ve fiziksel aktivite durumuna göre bireyselleştirilmektedir (2). Buna rağmen hastalarda günlük fiziksel aktiviteler ile eklem ve kas içi kanamalar nedeniyle kronik eklem hasarı gelişebilmektedir (3). Bu nedenle profilaksi tedavilerinin hastanın yaşam şekline göre aralıklarla tekrar düzenlenmesi önerilmektedir (1). Son yıllarda FVIII farmakokinetik (FK) verileri ile düzenlenen profilaksinin daha bașarılı olduğu bildirilmektedir (2). FVIII'in FK profilini hastaların yaş, kilo, von Willebrand faktör (VWF) düzeyi ve kan grupları etkilemektedir (4). Farmakokinetik profilin oluşturulması, bireysel FVIII dozunun belirlenmesinde, hastaların günlük aktivitelerinin düzenlenmesinde ve tedavi maliyetinin azaltılmasında en bașarılı stratejidir (4). Farmakokinetik çalıșmalarda elde edilen FVIII yarılanma ömrü (t1/2) ve FVIII çukur değerleri (bir sonraki FVIII konsantresi uygulamasından önceki düzey = trough level) profilaksi etkinliğini belirlemede kullanımaktadır (3). FVIII'in $<1$ IU/dL olduğu zaman dilimi spontan kanamalarla ilişkilidir (3). Sık kanayan, ağır fiziksel aktivite gösteren, aynı ekleminde sık kanama olan (hedef eklem) hastalarda daha yüksek çukur FVIII değerleri hedeflenlenmelidir (1). Bireysel olarak oluşturulan bu FK veriler ile hastaya göre FVIII dozu ve sıklığı belirlenmektedir.

Uluslararası Tromboz ve Hemostaz Topluluğu (International Society on Thrombosis and Haemostasis) standart yöntem olarak faktör almadan geçen 72 saat sonrasında erişkin hastalarda $9-11(0,0.25,0.5,1,3,6,9,24,28,32$, 48. saat), çocuklarda ise en az $5(0,1,9,24$, 48. saat) FVIII düzey ölçümü ile FK profil elde edilebileceğini bildirmektedir $(5,6)$. Çocuklarda FK verilerin hesaplanması için fazla sayıda örnek alımı hasta uyumsuzluğuna, okuldan uzak kalmaya, ebeveyn iş gücü kaybına neden olarak uygulamada güçlük oluşturmaktadır.
FVIII farmakokinetiğinin hesaplanmasında Bayesian modeli kolay uygulanması nedeniyle diğer yöntemlere göre üstündür (3). Bayesian metodu, büyük bir popülasyondan elde edilen FK veriler ile matematik modeli kullanılarak oluşturulmuştur (2). Hastaya ait az sayıdaki plazma FVIII düzey verisiyle hastanın FK verilerinin tahminini sağlamaktadır. Bu az örnekle yapılan tahminin standart yöntemlerle yüksek oranda benzer olduğu gösterilmiştir (6). Farmakokinetik ile uyarlanmış profilaksi rejimi yllık kanama sayısı, FVIII tüketimi ve yan etki açısından standart profilaksi rejiminden farklllik göstermemektedir (7). Farmakokinetik profil oluşturmak için Bayesian yöntemi kullanan farklı web programları bulunmaktadır (8). Bu programlar FK verilerin daha hızlı eldesini sağlarken maliyeti de düşürmektedir (9). Bu çalışmada hemofili A nedeniyle izlenen hastaların myPKFiT® (Takeda Pharmaceutical Company Limited, Lexington, MA, USA) ile FK verilerinin eldesi ve intiyaç duydukları FVIII dozlarının düzenlenmesi amaçlandı.

\section{GEREÇ VE YÖNTEMLER}

Bu çalışmaya Sağlık Bilimleri Üniversitesi, Ankara Çocuk Sağlığı ve Hastalıkları Hematoloji Onkoloji Sağlık Uygulama Araştırma Merkezi, Çocuk Hematoloji Onkoloji Kliniğinde Haziran 2017 Haziran 2018 tarihleri arasında ağır hemofili A tanısı ile izlenen primer veya sekonder profilaksi tedavisi gören çocuk veya erişkin yaştaki hastalar dahil edilmiştir. Hafif hemofili A nedeniyle izlenen veya iki yaşın altında olan, daha önceden bilinen FVIII inhibitörü olan hastalar çalışmaya dahil edilmemiştir. Hastaların demografik bilgileri, FVIII seviyesi ve inhibitör titreleri, F8 mutasyonu, FVIII profilaksi dozu, FVIII uygulama sıklığı kaydedilmiştir. Çalışma, Yerel Klinik Araştırmalar Etik Kurulu onayı ve tüm hasta ve/veya anne-babalarııı yazııı onamı alındıktan sonra gerçekleştirilmiştir ((Karar no: 2018-160).

Rekombinan FVIII (rFVIII) konsantresi [Advate® (Takeda Pharmaceutical Company Limited, Lexington, MA, USA)] tedavisi alan hemofili A hastalarında FK verilerin tahmininde myPKFiT® (Takeda Pharmaceutical Company Limited, Lexington, MA, USA) web erişimli yazlım programı (www.myPKFiT.com) kullanıldı. Test öncesi hastaların yaş, vücut ağırlığı, faktör arınma (en az 72 saat faktör konsantresi almaması) sonrası FVIII düzeyleri, uygulanan FVIII miktarı ve uygulama örnek alım saatleri myPKFiT® programına kaydedildi. Faktörden arınma sonrası 0. saat, 4. ve 24. saatlerde 3 kez plazma örnekleri alındı. Bu örneklerde FVIII düzeyi ve inhibitör testleri Gazi Üniversitesi Hastanesi Merkez Hematoloji-Hemostaz Laboratuvarı'nda Stago STA compact Max-2 coagulation analyzer ile çalışılı. 0-4 ve 24. saatte Faktör VIII aktiviteleri \% olarak dahil edildi. Bireysel 
FK profile örnek olarak, iki nolu hastaya ait veriler Şekil 1'de gösterilmiştir. Verilerin hesaplanması için Bayes algoritması (v2) kullanıldı. FK veri olarak FVIII'in klerensi, plazma yarılanma ömrü (FVIII t1/2), FVIII düzeyi \%1'in altına düşene kadar geçen zaman belirlendi. Hedef çukur değer olarak $>\% 1$ kabul edildi. Hedef çukur değer programa girildikten sonra bireysel haftalık doz takvimi simulasyon olarak elde edildi. Iki nolu hastaya ait elde edilen haftalık doz takvim simulasyonu örnek olarak Şekil 2'de gösterilmiştir. Hastanın yaşam şekline göre değiştirilen haftalık ilaç alım günleri hasta ve ailesiyle paylaşıldı.

Bu çalışmaya ağır hemofili $A$ tanısı ile primer veya sekonder profilaksi tedavisi alan, bilinen FVIII inhibitörü olmayan 19 erkek hasta dahil edildi. Hiçbir hastada daha önceden FK çalışma uygulanmamıştı. myPKFiT® ile FK çalışma planlanan iki hastanın biri yüksek titreli [>5 Bethesta ünitesi $(\mathrm{BU})]$ inhibitöre bağlı, diğerinin ise FVIII düzey sonuçları ile FK verilerin elde edilememesi nedeniyle çalışmadan çıkarıldı. Bir hastanın (3 no'lu hasta) test sırasında düşük titre (1.62 BU) FVIII inhibitörünün saptanması ve buna bağı FVIII yarılanma ömrünün 5.5 saat olması nedeniyle; FVIII yarılanma ömrü, klerens ve faktör düzeyinin <\%1 olmasına kadar geçen süre ortalamalarına dahil edilmedi. Üç hastada (7,8,11 no'lu hastalar) bireysel FK verilerin değişimini görmek açısından FK analizler iki kez yapıldı ve değerlendirmeye son yapılan sonuçları dahil edildi (Tablo I). Istatistik analizler The jamovi project (2019). jamovi. (Version
1.1) [Computer Software, Retrieved from https://www.jamovi. org] aracılığı ile yapıldı. Tanımlayıcı değişkenler ortanca veya ortalama olarak temsil edildi. Korelasyon analizi Spearman korelasyon analizi testi ile gerçekleştirildi. $\mathrm{P}<0.05$ istatistiksel anlamlı kabul edildi.

\section{BULGULAR}

Çalıșmaya dahil edilen 17 hastanın ortanca yașı 11.5 yıl (25p-75p: 7.8-16.9), ortanca vücut ağıllığı 32 kg (25p-75p: 2655.5), ortanca kullandıkları faktör miktarı $25 \mathrm{U} / \mathrm{kg}$ (25p-75p: 2028)'di. Beş hasta haftada iki, 12 hasta ise haftada üç gün FVIII ile profilaksi tedavisi almaktaydı. Genetik mutasyon testi yapılan 14 hastanın altısında (\%43) F8 geninde Intron-22 inversiyon mutasyonu bulunmaktaydı. Hastaların yaş, vücut ağırlığı, FVIII dozları ve sıklğı ve FK verileri Tablo l'de sunuldu. Ortalama FVIII yarlanma ömrü 9.1 saat (min-max: 6.8-11.4), ortalama klerens $4.7 \mathrm{ml} / \mathrm{st} / \mathrm{kg}$ (min-max: 2.6-6.8) ortalama FVIIl'in <\%1'e kadar geçen süre ise 47 saat (min-max: 33-61) olarak bulundu. Hasta yaşlarının artmasıyla FVIII yarılanma ömrü arttığı bulundu; pozitif yönlü orta düzey ilişki saptandı, Spearman r 0.496 ( $p=0.051$ ). Hasta yașlarının artmasıyla FVIII klerensi azalmaktaydl; negatif yönlü orta düzey ilişki saptandı, Spearman $r-0.426(p=0.1)$. Hastaların hedef çukur değeri \%1 üzeri olacak şekilde haftalık

Tablo I: Hastaların tanımlayıcı özellikleri ve farmakokinetik verileri.

\begin{tabular}{|c|c|c|c|c|c|c|c|c|c|c|}
\hline $\begin{array}{c}\text { Hasta } \\
\text { no }\end{array}$ & $\begin{array}{l}\text { Yaş } \\
\text { (yıl) }\end{array}$ & $\begin{array}{c}\text { Vücut } \\
\text { ağırlığı } \\
\text { (kg) }\end{array}$ & $\begin{array}{l}\text { FVIII dozu } \\
\text { (IU/kg) }\end{array}$ & F8 Mutasyon & $\begin{array}{c}\text { Doz } \\
\text { sıklığı } \\
\text { (/hf) }\end{array}$ & $t_{1 / 2}$ (st) & $\begin{array}{l}\text { Klerens } \\
\text { (ml/st/kg) }\end{array}$ & $\begin{array}{l}\text { \%1'e kadar } \\
\text { geçen süre } \\
\text { (st) }\end{array}$ & $\begin{array}{l}\text { İnhibitör } \\
\text { (BU) }\end{array}$ & $\begin{array}{c}\text { Doz } \\
\text { önerisi }\end{array}$ \\
\hline 1 & 25.0 & 60.0 & 25 & Bilinmiyor & 3 & 9 & 4.7 & 45 & - & Artır \\
\hline 2 & 15.0 & 37.0 & 27 & $\begin{array}{l}\text { c. } 1444-2 A>G \\
\text { (IVS9-2A>G) }\end{array}$ & 3 & 11.1 & 3.7 & 59 & - & Azalt \\
\hline 3 & 6.4 & 25.0 & 20 & c. $1804 \mathrm{C}>\mathrm{T}(\mathrm{R} 602 \mathrm{X})$ & 2 & 5.5 & 8.3 & 22 & 1.62 & Aynı \\
\hline 4 & 17.8 & 57.0 & 35 & 1967G>A (W656*) & 3 & 9.9 & 3.6 & 57 & - & Azalt \\
\hline 5 & 8.2 & 32.0 & 23 & Intron-22 inversiyon & 3 & 9.4 & 4.3 & 48 & - & Aynı \\
\hline 6 & 2.1 & 10.0 & 25 & Bilinmiyor & 2 & 6.8 & 6.8 & 34 & - & Aynı \\
\hline 7 & 8.7 & 27.0 & 19 & Intron-22 inversiyon & 2 & $\begin{array}{c}11.4 \\
9.3^{\star}\end{array}$ & $\begin{array}{l}2.6 \\
4.7^{\star}\end{array}$ & $\begin{array}{l}60 \\
44^{*}\end{array}$ & - & Artır \\
\hline 8 & 7.3 & 18.0 & 28 & $\begin{array}{l}\text { c. } 1715 \text { delT } \\
(\text { (1572Tfs } 7)\end{array}$ & 2 & $\begin{array}{l}7.8 \\
7.2^{*}\end{array}$ & $\begin{array}{l}4.8 \\
6.3^{*}\end{array}$ & $\begin{array}{l}40 \\
38^{*}\end{array}$ & - & Artır \\
\hline 9 & 11.5 & 27.0 & 28 & Intron-22 inversiyon & 3 & 10.1 & 3.8 & 55 & 0.66 & Azalt \\
\hline 10 & 16.1 & 65.0 & 23 & Bilinmiyor & 3 & 10.7 & 3.7 & 54 & - & Azalt \\
\hline 11 & 15.9 & 54.0 & 28 & c. $901 \mathrm{C}>\mathrm{T}(\mathrm{R} 301 \mathrm{C})$ & 3 & $\begin{array}{l}8.2 \\
7.8^{\star}\end{array}$ & $\begin{array}{l}5.2 \\
5.5^{\star}\end{array}$ & $\begin{array}{l}42 \\
40^{*}\end{array}$ & - & Artır \\
\hline 12 & 9.1 & 30.0 & 17 & Intron-22 inversiyon & 3 & 7.3 & 6 & 33 & - & Artır \\
\hline 13 & 8.7 & 30.0 & 25 & $6545 \mathrm{G}>\mathrm{A}(\mathrm{R} 2182 \mathrm{H})$ & 2 & 8 & 5.7 & 40 & 0.97 & Artır \\
\hline 14 & 17.6 & 50.0 & 20 & $6545 \mathrm{G}>\mathrm{A}(\mathrm{R} 2182 \mathrm{H})$ & 3 & 8.3 & 5.3 & 39 & - & Artır \\
\hline 15 & 19.1 & 70.0 & 29 & Intron-22 inversiyon & 3 & 11.2 & 3.9 & 61 & - & Azalt \\
\hline 16 & 3.9 & 14.5 & 17 & Intron-22 inversiyon & 3 & 7.9 & 5.6 & 36 & - & Artır \\
\hline 17 & 12.5 & 51.0 & 25 & $\begin{array}{l}\text { Hemizigot } \\
\text { c.201G>T (K67N) }\end{array}$ & 3 & 9.1 & 4.8 & 46 & 0.69 & Artır \\
\hline
\end{tabular}

*ilk yapılan myPKFiT analizi. 


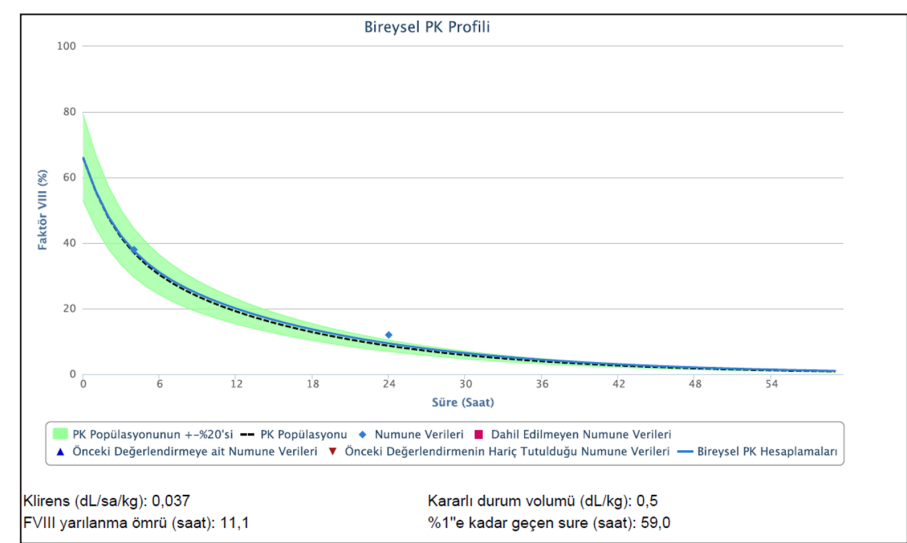

Şekil 1: İki nolu hastaya ait bireysel farmakokinetik profil. Verilerin hesaplanması için Bayes algoritması (v2) kullanılmıştır.

doz ve takvim simulasyonu uygulandığında 3 hastanın kullandığı FVIII dozunun yeterli olduğu, 5 hastada FVIII dozunun azaltılması gerektiği, 9 hastanın ise kullandığı FVIII dozunun yeterli olmadığı görüldü (Tablo I).

\section{TARTIȘMA}

Hemofili hastalarında faktör profilaksi tedavilerinin bireyselleștilmesi güncel yaklașım olarak önerilmektedir. Standart profilaksi hasta kilosu, toparlanma zamanı ve kanama fenotipine göre düzenlenmektedir. Ancak bu standart rejim bazı hastalarda yetersiz profilaksiye, korunmaya veya bazı hastalarda da gerekli olmayan ölçüde fazla doza (kaynak israfı) sebep olabilir $(1,3)$. Bu çalışmanın, merkezimizde daha önceden FK analiz yapılmamış hemofili A hastalarında profilaksinin bireyselleştirilmesine katkı sağladığı düşünülmektedir. Çalışmamızdaki hastaların yaklaşık üçte birinin fazla doz aldığı ve sağlık harcamaları maliyetini arttırdığı görülmüştür. Dokuz hastanın ise FVIII profilaksi dozunun yeterli olmadığı gösterilmiştir. Bu hastalarda kanama sayılarının fazla olması da sağlık harcamaları maliyetini artıracağı düşünülmektedir.

Çalıșmaya dahil edilen hastalardan 24 saat içinde 3 örnek alınarak FK verilerin eldesi hastalar açısından okula devamsızlık ve ebeveynlerin iş gücü kaybının en az olması, çocuklardan kan alma zorluğu gibi nedenlerden dolayı kolaylık sağlamıştır. Literatürde faktör uygulaması sonrası 2 örnek alınmasının da yeterli olabildiği bildirilmiştir (10). Collins PW. ve ark. (11) çocuklarda ortalama FVIII yarılanma ömrünü 9.3 saat, klerensini de $4.2 \mathrm{ml} / \mathrm{st} / \mathrm{kg}$ olarak bildirmiştir. Çalışmamızda da bu verilerle uyumlu olarak ortalama FVIII yarılanma ömrü 9.1 saat, ortalama klerens 4.7 ml/st/kg, ortalama FVIIl'in <\%1'e kadar geçen süre ise 47 saat olarak bulundu. Çalışmamızda çukur FVIII düzeyinin \%1'in altına düşmesi 48 saatten kısa süren hastalarda, FVIII düzeyi <\%1 olmasına karşın sık kanamamaları, trombin jenerasyonunu artıran telafi edici mekanizmalara işaret etmektedir (10). Yaş arttıkça FVIII yarılanma ömrü de artmaktadır (Spearman r 0.644) $(10,11)$. Çalışmamızda yaş

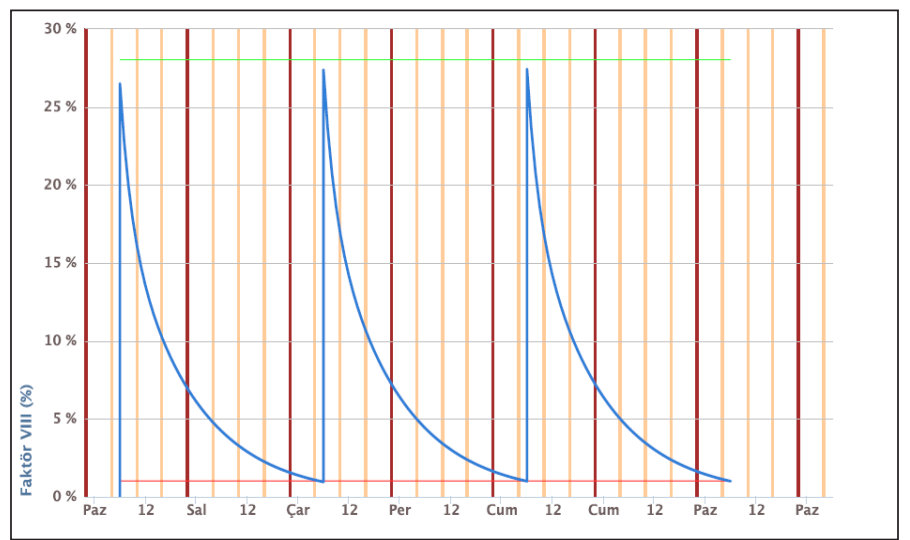

Şekil 2: İki nolu hastaya ait hedef çukur FVIII düzeyi \%1 olarak programa girildikten sonra elde edilen haftalık doz takvim simulasyonu.

ile FVIII yarılanma ömrü arasında yüksek oranda korelasyon saptanmaması, FVIII yarılanma ömrünün daha kısa olması beklenen 6 yaş altındaki hasta sayısının az olmasına bağlanmıştır. Farmakokinetik verilerin eldesinde myPKFiT yanında WebAccessible Population Pharmacokinetic Service-Hemophilia [WAPPS] ve NONMEM gibi Bayesian metodu kullanan diğer yöntemler de bulunmaktadır (8). Bu üç yöntemle farkı FK veriler elde edilebileceği ve farkı rFVIII profilaksi doz önerileri ortaya çıkabileceği de göz önünde bulundurulmalıdır (8).

Bayesian metod kullanılarak çocuk ve erişkinlerde FK veri elde edilen bir çalışmada hastaların yarısında profilaksi sıklığı ve dozlarında değișiklik yapılması gerekmiștir (12). Profilaksiye olan uyumda artış ve yıllık kanama sayısında azalma gösterilmiştir $(12$, 13). Hastalarımızda profilaksi rejim değişikliklerine karar verilirken FK profil ile birlikte bireysel kanama sıklıkları ve eklem sağlığına göre karar verildi. Farmakokinetik verilerle bireyselleştirilen profilaksi; standart, bireye göre düzenlenmemiş ve kanama riski yüksek olan rejimlerin optimize edilmesine, maliyetin azaltımasına katkı sağlamaktadır. Ayrıca hastanın eğitimine, günlük fiziksel aktivitelerinin ağırlığının ve gününün belirlenmesine, infüzyon programına olan uyumun (adherence) artmasına yol açmaktadır (2,3,10). myPKFiT'e göre rejim değişikliği önerilmeyen hastalarda ise uygulanan faktör doz ve sıklığının yeterli olduğu doğrulanmıştır. Hastaların FVıII düzeyinin \%1'in altında olduğu zamanın ve günlerin grafik olarak eldesi, hastaların bu zamanlarda aktivitelerini kısıtlayarak kanamalardan korunmayı sağlayacaktır. FK profile göre düzenlenen profilaksi dozu ve sıklığı, hastanın hayat kalitesinde artışa yol açacağı öngörülmektedir. Çalışmanın kısıtlııkları: hastaların hemofili eklem skorları, yıllık kanama sayıları dahil edilmemesi; az sayıda hastanın dahil edilmesi, hasta yaș aralıklarının geniş olması ve yapılan değişiklikler sonrası uzun dönem etkilerinin incelenmemesidir.

Sonuç olarak, myPKFiT®'in hasta eğitimine katkı sağlayan, tedavi uyumunu ve başarısını artıran, profilaksinin yönetimini kolaylaştıran bir araç olduğu düşünülmektedir. Ayrıca yıllık kanama sayısının ve maliyetin azalmasında potansiyel rolü 
bulunmaktadır. Daha geniş populasyonda, yaş grupları ayrıştıılarak, uzun dönem takip çalışmalarına intiyaç duyulmaktadır.

\section{KAYNAKLAR}

1. Petrini P, Valentino LA, Gringeri A, Re WM, Ewenstein B. Individualizing prophylaxis in hemophilia: a review. Expert Rev Hematol 2015;8:237-46

2. Pasca S, Milan M, Sarolo L, Zanon E. PK-driven prophylaxis versus standard prophylaxis: When a tailored treatment may be a real and achievable cost-saving approach in children with severe hemophilia A. Thromb Res 2017;157:58-63.

3. Megías-Vericat JE, Bonanad S, Haya S, Cid AR, Marqués MR, Monte E, Pérez-Alenda S, Bosch P, Querol F, Poveda JL. Bayesian pharmacokinetic-guided prophylaxis with recombinant factor VIII in severe or moderate haemophilia A. Thromb Res 2019;174:151-62.

4. McEneny-King A, lorio A, Foster G, Edginton AN. The use of pharmacokinetics in dose individualization of factor VIII in the treatment of hemophilia A. Expert Opin Drug Metab Toxicol 2016;12:1313-21.

5. Björkman S, Oh M, Spotts G, Schroth P, Fritsch S, Ewenstein BM, Casey $\mathrm{K}$, et al. Population pharmacokinetics of recombinant factor VIII: the relationships of pharmacokinetics to age and body weight. Blood 2012;119:612-8.

6. Björkman S. Limited blood sampling for pharmacokinetic dose tailoring of FVIII in the prophylactic treatment of haemophilia A. Haemophilia 2010;16:597-605.
7. Valentino LA, Mamonov V, Hellmann A, Quon DV, Chybicka A, Schroth $P$, et al. A randomized comparison of two prophylaxis regimens and a paired comparison of on-demand and prophylaxis treatments in hemophilia A management. J Thromb Haemost 2012;10:359-67.

8. Preijers T, van Moort I, Fijnvandraat K, Leebeek FWG, Cnossen MH, Mathôt RAA, et al. Cross-evaluation of Pharmacokinetic-Guided Dosing Tools for Factor VIII. Thromb Haemost 2018;118:514-25.

9. Pasca S, Zanon E. Savings without changing: How to use the

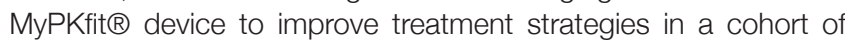
patients with haemophilia A. Thromb Res 2019;183:1-3.

10. Álvarez-Román MT, Fernandez-Bello I, de la Corte-Rodríguez $\mathrm{H}$, Hernández-Moreno AL, Martín-Salces M, Butta-Coll N, et al. Experience of tailoring prophylaxis using factor VIII pharmacokinetic parameters estimated with myPKFiT( $(\Omega)$ in patients with severe haemophilia A without inhibitors. Haemophilia 2017;23:e50-e54.

11. Collins PW, Blanchette VS, Fischer K, S Björkman, M Oh, S Fritsch, et al. Break-through bleeding in relation to predicted factor VIII levels in patients receiving prophylactic treatment for severe hemophilia A. J Thromb Haemost 2009;7:413-420.

12. Nagao A, Yeung CHT, Germini F, Suzuki T. Clinical outcomes in hemophilia A patients undergoing tailoring of prophylaxis based on population-based pharmacokinetic dosing. Thromb Res 2019;173:79-84

13. Mingot-Castellano ME, Parra R, Núñez R, Martorell M. Improvement in clinical outcomes and replacement factor VIII use in patients with haemophilia A after factor VIII pharmacokinetic-guided prophylaxis based on Bayesian models with myPKFiT(®). Haemophilia 2018;24:e338-e343. 